\title{
Factores de riesgo para mortalidad en neumonía asociada a ventilación mecánica
}

\author{
Osvaldo Iribarren B., Jacquelin Aranda T., Lilian Dorn H., Mónica Ferrada M., \\ Héctor Ugarte E., Vinka Koscina M., Daniel López R. y Mauro Morel F.
}

\section{Mortality risk factors in ventilator associated pneumonia}

Objective: to identify lethality and mortality rates and, mortality risk factors in ventilator associated pneumonia (VAP) on 114 patients treated between 2000 and 2007. Method: Twenty five risk factors were analyzed, emphasizing age, gender, APACHE score, associated diseases, hypotension at intake, coma, hospitalization time, length of time of ventilation, emergency intubation, reintubation, previous antibiotics, and resistant microrganisms. Results: Lethality was $25.4 \%$, and mortality was $2.4 \%$. Association between lethality, and APACHE score was found (p: 0.04). Critical APACHE value was 22. Also, in early pneumonia, association between lethality and nasogastric tube (p: 0.01 , I.C. $95 \% 1.39-6.35$ ) was found. No association with late pneumonia was found among mortality and clinical practices. Death's RR (relative risk) increase in following values with: previous neurological disease 2.7 (p: 0.15, IC $95 \% 1.15-6.5$ ), neurological coma RR 2 (p: 0.2 , IC $95 \% 0.54-7.53$ ). Nevertheless, at multivariate analysis no mortality risk factors were identified. Fair association with time in ICU (p: 0.051 IC $95 \% 0.99-1.17$ ) and, male sex (p: 0.051 , IC $95 \% 0.99$ - 6.72) was found. Conclusions: We observed multiple factors associated to mortality in VAP: use of nasogastric catheter, longer stay in ICU and male sex.

Key words: Pneumonia ventilator associated, mechanical ventilation, nosocomial infection.

Palabras clave: Neumonía asociada a ventilación mecánica, ventilación mecánica, infección intrahospitalaria.

\section{Introducción}

$\mathrm{L}$ a neumonía asociada a ventilación mecánica (NAVM) es el mayor riesgo que enfrentan los pacientes conectados a asistencia ventilatoria mecánica, la infección de mayor prevalencia en las unidades de cuidados intensivos ${ }^{1}$ y la principal causa de muerte por infección intrahospitalaria ${ }^{2}$. La letalidad de los pacientes ventilados por más de 48 horas es de $\sim 20$ a $25 \%$, con una incidencia de $1 \%$ adicional por cada día de ventilación mecánica (VM). Se estima que el riesgo de adquirir neumonía es 21 veces mayor en los pacientes expuestos a VM, comparados con los pacientes no sometidos al procedimiento. La mortalidad adicional que provoca la NAVM, o mortalidad atribuible, ha sido estudiada observándose un amplio rango que va desde 30 a $70 \%$, según diferentes estu$\operatorname{dios}^{3-5}$. Por otra parte, estos y otros reportes muestran que en los sobrevivientes se prolonga significativamente la estadía hospitalaria entre 19 y 44 días $^{6}$.

El mecanismo principal en la patogenia de la NAVM es la micro-aspiración repetida de microorganismos que colonizan las vías aéreas superiores, a través del espacio comprendido entre el balón del tubo endo- traqueal y la pared de la tráquea. La procedencia de estos microorganismos varía entre la microbiota endógena del paciente y los bacilos gramnegativos no fermentadores de fuentes ambientales, principalmente las manos del personal sanitario o los nebulizadores contaminados $^{7-9}$.

Los países latinoamericanos presentan importantes diferencias cuando se comparan con las naciones desarrolladas en características genéticas, calidad de los servicios de salud o desarrollo en investigación clíni$\mathrm{ca}^{8}$. En esas condiciones, se puede establecer la hipótesis que la incidencia y factores de riesgo para el desarrollo de NAVM puede ser diferente a la que se describe en los países del primer mundo. Nuestro hospital cuenta con un programa y personal de vigilancia epidemiológica con dedicación exclusiva desde 1999. Excepto el uso de la técnica aséptica y el lavado de manos, no hemos evaluado otros factores de riesgo asociados a los pacientes y a las prácticas clínicas que puedan ser intervenidos para reducir esta conocida complicación de la VM.

El objetivo de este estudio es identificar la tasa de letalidad, la tasa de mortalidad y los factores de riesgo de mortalidad asociados a NAVM.

\author{
Hospital Clínico San Pablo de \\ Coquimbo, Chile \\ Comité de IIH (OIB, JAT, LDH, MFM) \\ Unidad de Cuidados Intensivos (HUE) \\ Universidad Católica del Norte, \\ Chile \\ Escuela de Medicina (VKM, DLR,
} MMF)

Recibido: 23 de septiembre 2008 Aceptado: 22 de enero 2009

Establecimiento donde se realizó el estudio: Hospital San Pablo de Coquimbo.

Financiamiento: Los autores Los autores declaran no tener conflicto de intereses

Correspondencia a: Osvaldo Iribarren Brown oiribarren@123.cl 


\section{Pacientes y Métodos}

Se incluyeron en el presente estudio todos los pacientes con el diagnóstico de NAVM atendidos en la UCI del Hospital San Pablo de Coquimbo de la cohorte 2000-2007, y que fueron sometidos a vigilancia epidemiológica selectiva y prospectiva durante el tiempo que permanecieron hospitalizados.

Tipo de estudio: Cohorte longitudinal observacional.

\section{Objetivos}

- Describir la incidencia, factores de riesgo, y desenlace de la NAVM en una cohorte de población no seleccionada en la Unidad de Cuidados Intensivos del Hospital San Pablo de Coquimbo.

- Identificar la tasa de mortalidad y letalidad de la NAVM

- Comparar el desenlace entre los pacientes con neumonía de aparición precoz y neumonía de aparición tardía.

- Identificar los microorganismos aislados de acuerdo al tiempo de presentación de la enfermedad.

- Identificar la tasa de letalidad, de acuerdo a la susceptibilidad in vitro de los microorganismos, considerando especies sensibles y resistentes a antimicrobianos.

Criterios de inclusión ${ }^{9-11}$ :

- Pacientes con intubación endo-traqueal y sometidos a VM por un período superior a 48 horas.

- Edad mayor de 15 años.

- Estadía en UCI por un período superior a 48 horas.

- Padecimiento de NAVM. Se incluyeron pacientes con uno o más episodios de NAVM.

Criterios de exclusión:

- Pacientes sin confirmación de NAVM.

- Neumonía intra-hospitalaria no asociada a VM.

- Pacientes con traqueostomía previa a la NAVM.

Definiciones. Neumonía: infiltrado pulmonar radiológico nuevo o progresivo más dos de los siguientes criterios: $\mathrm{T}^{\circ}$ axilar de $>38^{\circ} \mathrm{C}$ ó $<36{ }^{\circ} \mathrm{C}$, secreción traqueal purulenta, recuento de leucocitos en sangre mayor de $10.000 / \mathrm{mm}^{3}$ y aislamiento de bacterias patógenas del aspirado endotraqueal ${ }^{2,11-13}$. Se utilizó la metodología de cultivo cuantitativo de aspirado endotraqueal y el nivel de corte utilizado fue de $10^{5}$ colonias $/ \mathrm{ml}^{311,13}$. Neumonía asociada a ventilación mecánica: neumonía en paciente con intubación endotraqueal y sometido a ventilación mecánica por un período mayor a 48 horas. Neumonía precoz y neumonía tardía: ocurrida en pacientes con menos de cuatro días de VM y si ocurrió después de este período respectivamente ${ }^{12,14}$. Microorganismos resistentes a antimicrobianos: Staphylococcus aureus resistente a meticilina ${ }^{15,16}$, bacilos gramnegativos resistentes a cefalosporinas de $3^{\text {a }}$ generación (ceftriaxona, cefotaxima) ${ }^{17}$. El puntaje APACHE (Acute Physiology and Chronic Health Evaluation) fue calculado según criterios previamente establecidos, a partir de los datos clínicos disponibles ${ }^{18}$. Este puntaje clasifica la gravedad de la enfermedad, con independencia del diagnóstico y predice la evolución de los pacientes. Antimicrobiano previo: tratamiento con antimicrobianos durante su estada en el hospital y antes del diagnóstico de NAVM. Mortalidad por 1.000 días de exposición: cuociente entre el número de fallecidos y 1.000 días de exposición al factor de riesgo.

Base de datos y análisis estadístico: Recolección y procesamiento de datos en programa EPI INFO 3.5. Los factores de riesgo ${ }^{2,3,8,9,14,19}$ para el desarrollo de NAVM estudiados fueron los siguientes:

- Del paciente: Edad, sexo, gravedad de la enfermedad al ingreso (puntaje APACHE), gravedad de la enfermedad al momento del diagnóstico de neumonía (puntaje APACHE), origen, número de enfermedades asociadas, presencia de hipotensión arterial y/o de coma al ingreso.

- De la atención: Duración de la hospitalización, duración de la VM, intubación de emergencia, reintubación, uso de antimicrobianos previos, presencia de microorganismos multi-resistentes.

Las tasas de referencia usadas en el estudio corresponden al percentil 75 de la incidencia de NAVM en Chile para cada año, publicados por MINSAL ${ }^{20,21}$. Las muertes fueron confirmadas en el Servicio de Registro Civil e Identificación. Se utilizó un análisis univariado para comparar las variables de los grupos con distinto desenlace. Todas las dócimas de significancia se obtuvieron con dos colas. Las variables cuantitativas se compararon utilizando la $t$ de Student para variables distribuidas normalmente. Se utilizó la prueba estadística de $\chi^{2}$ o la prueba exacta de Fischer para comparar variables categóricas. Se definió el valor de significancia estadística $p<0,05$.

Para el análisis multivariado se utilizó regresión logística y análisis multivariado NMDS (non metric multidimentional scaling), que utiliza rangos de similitud y tendencias (algoritmo de Sheppard-Kruskal), considerando como índice la distancia euclidiana de una matriz triangular simétrica ${ }^{22}$.

\section{Resultados}

En el período de estudio (ocho años), la vigilancia epidemiológica notificó 1.202 pacientes expuestos a VM por más de 48 horas y 174 casos presentaron neumonía, lo que entregó una tasa de incidencia glo- 


\begin{tabular}{|c|c|c|c|c|c|c|c|c|c|}
\hline Año & 2000 & 2001 & 2002 & 2003 & 2004 & 2005 & 2006 & 2007 & Total \\
\hline Número de pacientes expuestos & 116 & 155 & 116 & 143 & 139 & 164 & 172 & 197 & 1.202 \\
\hline Número de días en ventilación mecánica & 473 & 697 & 522 & 607 & 617 & 713 & 1149 & 849 & 5.627 \\
\hline Número de neumonías & 25 & 22 & 17 & 26 & 23 & 18 & 21 & 22 & 174 \\
\hline Tasa x 1.000 días exposición & 52,8 & 31,6 & 32,6 & 42,8 & 37,2 & 25,2 & 18,3 & 26,1 & \\
\hline Referencia nacional ( $p$ 75) & 49 & 49 & 32 & 32 & 29 & 29 & 26 & 25 & \\
\hline
\end{tabular}

bal de NAVM de $14,5 \%$. La muestra fue de 114 pacientes, ya que en 60 pacientes la historia clínica estaba incompleta $\mathrm{y}$, esos casos fueron descartados del estudio. Se identificaron 29 fallecidos con una tasa de letalidad de $25,4 \%$ y una tasa de mortalidad por exposición a VM de 2,4\% (Tabla 1). Las tasas anuales de neumonía por 1.000 días de exposición durante el período muestran una tendencia decreciente.

Las enfermedades que motivaron el ingreso a la UCI en los 114 pacientes fueron: intervenciones neuroquirúrgicas complejas (n: 27), traumas múltiples (n: 17), accidentes vasculares cerebrales (n: 17), traumatismo encéfalo craneano (n: 10), grandes quemados (sin quemadura de vía aérea) (n: 10), pancreatitis (n: 10), trauma cerrado (n: 8), infecciones abdominales (n: 9) e infecciones de otro origen (n: 6).

Se presentan los factores demográficos de los pacientes con neumonía precoz y tardía: edad, sexo, número de diagnósticos asociados, puntaje APACHE, presencia de enfermedad pulmonar obstructiva (Tabla 2).

Al evaluar la asociación entre factores de riesgo del paciente y las prácticas clínicas para variables cuantitativas, sólo se encontró una asociación significativa entre el puntaje APACHE al momento del diagnóstico de neumonía y la mortalidad, (p: 0,04). El valor crítico de APACHE fue de 22. Para las otras variables no se identificó una asociación estadística.

Para las variables categóricas no se encontró asociación significativa entre la exposición y mortalidad (Tablas 2 y 3). Sin embargo, el RR en presencia de enfermedad neurológica previa (A.V.E.) es de 2,7 y, coma al ingreso de 2. Para neumonía de instalación precoz el uso de fármacos vasoactivos y antimicrobianos previos aumenta el RR a 2,2 y el riesgo atribuible al uso de antimicrobianos es de 1,33.

Al estratificar a los pacientes en portadores de neumonía precoz (n: 60) y neumonía tardía (n: 54), en el grupo de neumonía precoz se identificó como factor de riesgo de muerte, la presencia de sonda naso-gástrica (p: 0,01, IC $95 \%$ 1,39-6,35), con un riesgo atribuible de 0,49 . En el grupo portador de neumonía de aparición tardía, no se identificaron factores de riesgo asociados a mortalidad (Tabla 4).
Tabla 2. Factores de riesgo de mortalidad. Neumonía asociada a ventilación mecánica. UCI Hospital San Pablo de Coquimbo, Chile. 2000-2007

\begin{tabular}{|lccccccc|} 
Variables & $\begin{array}{c}\text { Media } \\
\text { Vivos }\end{array}$ & $\begin{array}{c}\text { Media } \\
\text { Muertos }\end{array}$ & DS Student & P \\
\hline Edad & 47,5 & 18 & 50,8 & 18,5 & 0,87 & 0,38 \\
\hline Días de estada & 21,9 & 16,2 & 17 & 8,9 & 1,53 & 0,12 \\
\hline Días de ventilación mecánica & 15,1 & 16,6 & 13 & 7,9 & 0,66 & 0,51 \\
\hline Puntaje APACHE al ingreso & 20,3 & 6,7 & 20,2 & 6,5 & 0,07 & 0,94 \\
\hline Puntaje APACHE al diagnóstico de NAVM & 19,1 & 5,9 & 21,8 & 6 & 2,05 & 0,04 \\
\hline Co-morbilidad & 0,94 & 1,35 & 0,82 & 0,88 & 0,08 & 0,93 \\
\hline Intervalo intubación/ventilación & 1,5 & 0,6 & 1,5 & 0,41 & 0,9 & 0,36 \\
\hline NAVM: neumonía asociada a ventilación mecánica & & & & & \\
\hline
\end{tabular}

Tabla 3. Factores de riesgo de mortalidad en neumonía asociada a ventilación mecánica. UCI Hospital San Pablo de Coquimbo, Chile. 2000-2007

\begin{tabular}{|c|c|c|}
\hline Variables & P (IC 95\%) & $\mathbf{R} \mathbf{R}$ \\
\hline Sexo femenino & $0,08(0,91-3,13)$ & 1,69 \\
\hline Antimicrobianos previos & $0,57(0,49-2,16)$ & 1,03 \\
\hline Catéter vesical & $0,09(0,04-1,81)$ & 0,26 \\
\hline Fármacos vasoactivos & $0,24(0,7-2,75)$ & 1,39 \\
\hline Catéter venoso central & $0,58(0,38-2,93)$ & 1,05 \\
\hline Sonda naso-gástrica & $0,15(0,8-2,96)$ & 1,54 \\
\hline Intubación de emergencia & $0,28(0,67-2,49)$ & 1,3 \\
\hline Re-intubación & $0,35(0,42-1,56)$ & 0,81 \\
\hline Intubación nasal & $0,5 \quad(0,43-1,9)$ & 0,91 \\
\hline Alimentación enteral & $0,56(0,31-2,52)$ & 0,88 \\
\hline Hipertensión arterial & $0,31(0,66-2,36)$ & 1,25 \\
\hline Diabetes mellitus & $0,43(0,35-1,9)$ & 0,82 \\
\hline Enfermedad neurológica & $0,15(1,15-6,5)$ & 2,7 \\
\hline EPOC & $0,29(0,43-1,9)$ & 0,67 \\
\hline Hipotensión arterial al ingreso & $0,56(0,53-1,59)$ & 1,02 \\
\hline Coma al ingreso & $0,2 \quad(0,54-7,53)$ & 2 \\
\hline Neumonía precoz & $0,51 \quad(0,56-1,99)$ & 1 \\
\hline Neumonía tardía & $0,51 \quad(0,50-1,90)$ & 1 \\
\hline
\end{tabular}


Tabla 4. Factores de riesgo de la atención. Mortalidad por neumonía precoz y tardía. UCI Hospital San Pablo de Coquimbo, Chile. 2000-2007

\begin{tabular}{llllll} 
Variables & $\begin{array}{c}\text { Neumonía precoz } \\
\text { P (IC 95\%) }\end{array}$ & $\mathbf{R R}$ & $\begin{array}{c}\text { Neumonía tardía } \\
\text { P (IC 95\%) }\end{array}$ & R R \\
\hline Intubación de emergencia & $0,50(0,47-2,72)$ & 1,14 & $0,58(0,34-2,57)$ & 0,92 \\
\hline Intubación nasal & $0,48(0,45-3,04)$ & 1,17 & $0,49(0,25-2,46)$ & 0,79 \\
\hline Reintubación & $0,48(0,49-2,64)$ & 1,14 & $0,14(0,14-1,48)$ & 0,46 \\
\hline Alimentación enteral & $0,38(0,07-3,19)$ & 0,49 & $0,37(0,5-5,5)$ & 1,67 \\
\hline Antimicrobianos previos & $0,61(0,26-3,3)$ & 0,94 & $0,6(0,39-2,7)$ & 2,25 \\
\hline Fármacos vasoactivos & $0,51(0,45-2,74)$ & 1,12 & $0,16(0,85-5,92)$ & 2,25 \\
\hline Bloqueadores H2 & $0,62(0,34-2,9)$ & 1,0 & $0,7(0,39-2,7)$ & 1,0 \\
\hline Sonda naso-gástrica & $0,01(1,3-3,7)$ & 3,0 & $1,3(1,15-2,3)$ & 1,5 \\
\hline Catéter venoso & $0,61(0,3-3,7)$ & 1,0 & $0,59(0,25-7,1)$ & 1,33 \\
\hline
\end{tabular}

\begin{tabular}{|c|c|c|c|c|}
\hline & Odds ratio & LI $95 \%$ & LS $95 \%$ & p value \\
\hline Días estada en UCl & 1,084 & 0,99 & 1,17 & 0,051 \\
\hline Duración de la ventilación mecánica & 0,936 & 0,86 & 1,01 & 0,11 \\
\hline Edad & 0,985 & 0,96 & 1,01 & 0,28 \\
\hline Sexo masculino/femenino & 2,584 & 0,99 & 6,72 & 0,051 \\
\hline Uso sonda naso-gástrica & 2,172 & 0,74 & 6,3 & 0,15 \\
\hline Alimentación enteral & 0,93 & 0,18 & 4,75 & 0,93 \\
\hline Catéter venoso & 1,35 & 0,25 & 7,12 & 0,72 \\
\hline Antimicrobianos previos & 0,96 & 0,84 & 1,10 & 0,6 \\
\hline
\end{tabular}

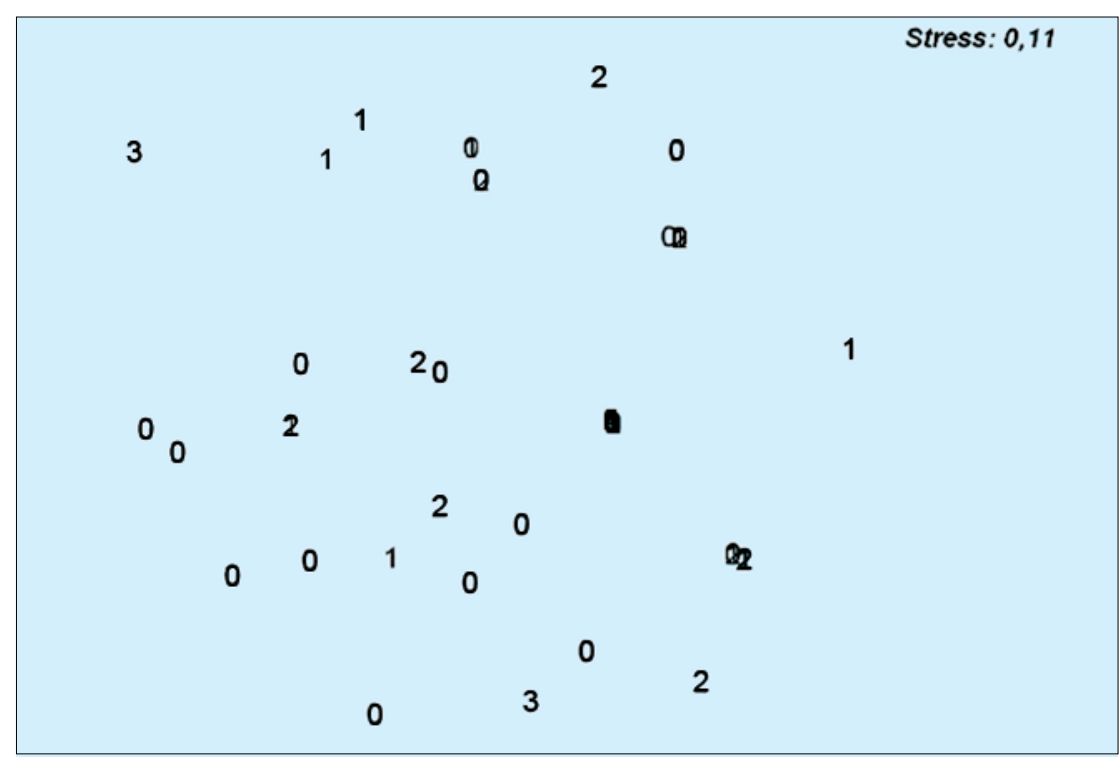

Figura 1. Co-morbilidad asociada a mortalidad en NAVM. Análisis multivariado NMDS. Hospital San Pablo de Coquimbo 2000-2007. 0: Sin co-morbilidad, 1: Una enfermedad asociada, 2: Dos enfermedades asociadas, 3: Tres enfermedades asociadas.
El análisis multivariado mediante regresión logistica, para definir la probabilidad de muerte asociada a la exposición días de estada en UCI, días de VM, sexo de los pacientes, presencia de $\mathrm{CVC}$, sonda naso-gástrica, alimentación enteral y uso de antimicrobianos previos, no mostró asociación significativa alguna (Tabla 5).

El análisis mediante NMDS para evaluar asociación entre co-morbilidad, categorizada según el número de enfermedades preexistentes (diabetes, mellitus insuficiencia renal, enfermedad pulmonar, hipertensión arterial) y mortalidad, mostró que no hay agrupamiento de datos y por lo tanto, no hay asociación entre comorbilidad (número de enfermedades previas) y el desenlace de los pacientes (Figura 1).

Los microorganismos aislados más frecuentemente en el aspirado bronquial fueron Acinetobacter baumannii 63/114 casos (55,2\%), Pseudomonas aeruginosa 31/114 casos (27,2\%), Klebsiella pneumoniae 28/114 casos (24,6\%), Staphylococcus aureus meticilina sensible (SAMS) 38/114 casos (33,3\%), y Staphylococcus aureus meticilina resistente (SAMR) en $7 / 114$ casos $(6,1 \%)$. En 78 casos $(68,4 \%)$ el cultivo fue polimicrobiano; la asociación más frecuente fue Acinetobacter baumannii -Staphylococcus aureus.

En neumonía de aparición precoz las especies más frecuentes fueron: A. baumannii en 36/60 (60\%) y SAMS en $22 / 60(36,6 \%)$. En neumonía de aparición tardía los microorganismos más frecuentes fueron: $A$. baumannii en 27/51 (52,9\%) y Pseudomonas aeruginosa en $18 / 51$ casos $(35,3 \%)$. En tres casos no se identificó la etiología.

La letalidad de las diversas cepas identificadas fue: SAMS $28,9 \%$, SAMR $42,9 \%, A$. baumannii $28,1 \%, A$. baumannii multi-resistente 50\%, $P$. aeruginosa $6,4 \%$, $P$. aeruginosa multi-resistente $20 \%$. Sin embargo, no se encontró significación estadística para dicha letalidad.

\section{Discusión}

La NAVM es una complicación que ocurre en alrededor de 20 a $25 \%$ de los pacientes ventilados durante más de 48 horas, con una incidencia de 1\% adicional por cada día de $\mathrm{VM}^{1}$. Nuestra serie muestra una incidencia menor, de $14,5 \%$, que se puede explicar por la reducción progresiva de la incidencia de esta complicación, a lo largo de los ocho años del estudio, generada por múltiples intervenciones de capacitación y mejoras técnicas recomendadas por el comité de prevención de infecciones intrahospitalarias: adherencia a la técnica aséptica en intubación y aspiración, lavado de manos, aislamiento de contacto en presencia de microorganismos multi-resistentes, cambio de circuitos entre pacientes, uso de agua estéril en humidificadores ${ }^{23}$. 
En ocho años de observación, la tasa de NAVM por 1.000 días de exposición se ha reducido a la mitad. Nuestra letalidad de $25,4 \%$ es similar a los valores nacionales de referencia en adultos, que muestran una letalidad de $26 \%{ }^{11}$. En nuestra serie, el riesgo de morir se asocia significativamente al uso de sonda nasogástrica. El RR se duplica en los pacientes más graves (coma al ingreso, uso de fármacos vasoactivos, enfermedades previas, APACHE igual o mayor de 22 al momento diagnóstico de neumonía) condiciones del hospedero en que no es posible intervenir.

De acuerdo a los resultados, algunas prácticas clínicas deben ser restringidas y racionalizadas para reducir el riesgo asociado a su uso, como la aspiración asociada al uso de sonda naso-gástrica. La asociación entre el uso previo de antimicrobianos y el desarrollo de microorganismos multi-resistentes no fue estudiada específicamente. Sin embargo, el RR de muerte por neumonía tardía fue 2,2 en presencia de antimicrobianos previos (p: NS). Nuestros resultados son similares a factores de riesgo individualizados en otros estudios $^{24}$ en que, alimentación por sonda nasogástrica, uso previo de antimicrobianos y re-intubación son considerados factores de riesgo para NAVM.

Los resultados sugeridos de mayor riesgo de muerte en el grupo de pacientes portadores de neumonía precoz que portan sonda naso-gástrica no se confirman con el análisis multivariado. Sin embargo, hay una asociación débil entre mayor permanencia del paciente en UCI y pacientes de sexo masculino. Tales resultados sugieren mortalidad mayor en pacientes más graves, que naturalmente tienen mayor estadía y pacientes hombres, en que hay mayor incidencia de trauma grave. Para definir el rol de la sonda naso-gástrica se requiere un estudio futuro en que específicamente se constate el desenlace en grupos comparables en que se utilice y no se utilice ese procedimiento.

La frecuencia de microorganismos aislados en la NAVM varía en las diversas publicaciones ${ }^{25-28}$, destacando SAMS y SAMR. Las neumonías tardías, generalmente se asocian a especies intra-hospitalarias ${ }^{26}$. Nuestra serie muestra que los microorganismos más frecuente en neumonía precoz y tardía son $A$. baumannii y $P$. aeruginosa. Esta realidad sugiere que la colonización de nuestros pacientes ha estado asociada a quiebres en las prácticas clínicas, particularmente en el uso de técnica aséptica. Por otra parte, cobra importancia la necesidad de reducir al máximo la exposición de los pacientes a antimicrobianos de amplio espectro para disminuir la presión antimicrobiana sobre las bacterias ${ }^{28}$. Finalmente, nuestros resultados muestran que la letalidad fue más frecuente en presencia de cepas multi-resistentes. Estas cepas se asocian a estadía prolongadas, a tratamientos iniciales que no dan cobertura contra los microorganismos causales de la neumonía, a mayor letalidad que las cepas sensibles y mortalidad atribuible considerable ${ }^{30,31}$. Nuestra experiencia comparte esa realidad por lo que la terapia empírica debe basarse en el perfil etiológico local y sus patrones de resistencia ${ }^{32}$.

En conclusión, nuestros resultados muestran una relación multifactorial de las prácticas clínicas y aspectos propios del paciente para el desarrollo de NAVM. Aquellos factores asociados a las características del paciente que ingresa a VM no son modificables. Sin embargo, los factores de riesgo asociados a las prácticas clínicas nos ha estimulado a desarrollar estrategias en ese ámbito que reduzcan esa exposición y han abierto una nueva línea de investigación que permita medir el impacto de procedimientos en el desarrollo de NAVM, como el uso de la sonda naso-gástrica.

Agradecimientos: A Domingo Lancelotti, Bioestadístico, por la revisión e interpretación estadística de los resultados y a Muriel Ramírez, Especialista en Salud Pública, de nuestra Facultad de Medicina, por la revisión del manuscrito.

\section{Resumen}

Objetivo: Identificar la tasa de letalidad, mortalidad y factores de riesgo de mortalidad en neumonía asociada a ventilación mecánica (NAVM) en 114 pacientes de la cohorte 2000-2007. Se estudiaron 25 factores de riesgo del paciente y de las prácticas clínicas. Resultados: La tasa de letalidad fue $25,4 \%$ y la tasa de mortalidad de 2,4\%. Se encontró asociación entre el puntaje APACHE al momento del diagnóstico de neumonía y mortalidad (p: 0,04). El valor crítico de APACHE de alto riesgo fue igual o mayor a 22. En neumonía precoz se identificó como factor de letalidad la presencia de sonda naso-gástrica (p: 0,01, IC 95\% 1,39-6,35). Para las variables categóricas no se encontró asociación significativa entre la exposición y mortalidad. El RR en presencia de enfermedad neurológica previa (accidente vascular encefálico) fue 2,7 (p: 0,15, IC 95\% 1,15-6,5), coma al ingreso 2 (p: 0,2, IC 95\% 0,54-7,53). En neumonía tardía, no se identificaron factores de riesgo asociados a la atención. El análisis multivariado de todas esas exposiciones no identificó factores significativos asociados a mortalidad. Identificamos una asociación débil con días de estada en UCI (p: 0,051 IC $95 \% 0,99-1,17$ ) y sexo masculino (p: 0,051 , IC 95\% 0,996,72). Conclusiones: Los resultados muestran una relación multifactorial de prácticas clínicas y del paciente para fallecer por NAVM. Como factor de prácticas clínicas encontramos asociado a mortalidad el uso de sonda nasogástrica y mayor permanencia en UCI. Dependiente del paciente encontramos una débil asociación entre mortalidad y sexo masculino. 


\section{Referencias}

1.- Woske J, Röding T, Schulz I, Lode H. Ventilator-associated pneumonia in a surgical intensive care unit: epidemiology, etiology and comparison of three bronchoscopic methods for microbiological specimen sampling. Crit Care 2001; 5: 167-73.

2.- Hugues G, Leroy O, Guery B, Alfandari S, Beaucaire G, Predisposing factors for nosocomial pneumonia in patients receiving mechanical ventilation and requiring tracheotomy. Chest 2000; 118: 767-74.

3.- Arancibia H F, Fica C A, Herve E B, Ruiz M, Yunge M. Diagnóstico de neumonía asociada a ventilación mecánica. Rev Chil Infect 2001; 18 (Supl 2): 41-57.

4.- Torres A, El-Ebiary M. Diagnostic approaches and hospital-acquired pneumonia. Sem Respir Crit Care Med 1997; 18: 149-61.

5.- Fagon J Y, Chastre J, Hance A J, Montravers P, Novara A, Gibert C. Nosocomial pneumonia in ventilated patients: a cohort study evaluating attributable mortality and hospital stay. Am J Med 1993; 94: 281-8.

6.- Brenner P, Nercelles P, Pohlenz M, Otaíza F. Costo de las infecciones intrahospitalarias en hospitales chilenos de alta y mediana complejidad. Rev Chil Infect 2003; 20: 285-90.

7.- Maciquez R, Castro B, Machado O, Manresa D. Neumonía nosocomial asociada a ventilación mecánica. Rev Cubana Pediatr 2002; 74: 222-32.

8.- Bergmans D, Bonten M. Nosocomial pneumonia. En Mayhall G: Hospital Epidemiology \& Infection Control. Lippincott, Williams \& Wilkins 2004. Third ed: 311-39.

9.- Noor A, Hussain F. Risk factors associated with development of ventilator associated pneumonia. J Coll Physicians Surg Pak 2005; 15: 92-5.

10.- Ylipalosaari P, Ala-Kokko T I, Laurila J, Ohtonen P, Syrjälä H. Epidemiology of intensive care unit (ICU)-acquired infections in a 14-month prospective cohort study in a single mixed Scandinavian university hospital ICU. Acta Anaesthesiol Scand 2006; 50: 1192-7.

11. - Labarca L J. Neumonía asociada a ventilación mecánica Rev Chil Infect 2001; 18 (Supl 2): 39-40.

12.- Trucco O. Neumonía asociada a ventilación mecánica: lavado broncoalveolar y urea en recuento bacteriano. Rev Chil Infect 2005; 22: 370.

13.- Klompas M: Does this patient have ventilator-associated pneumonia? JAMA 2007; 297: 1583-93.

14. - Ibrahim E, Ward S, Sherman G, Kollef M H, A Comparative analysis of patients with early-onset $v s$ late-onset nosocomial pneumonia in the ICU setting. Chest 2000; 117: 1434-42

15.- Raymond D, Pelletier S, CrabtreeT, Evans E, Pruett T, Sawyer T. Impact of antibiotic resistant Gram negative bacilli infection on outcome of hospitalized patients. Crit Care Med 2003; 31: 1035-41.

16.- Daum R, Seal J. Evolving antimicrobial chemotherapy for Staphylococcus aureus infections: our backs to the wall. Crit Care Med 2001; 29 Suppl: N 92-6.

17.- Rupp M E, Fey P D. Extended spectrum beta lactamase producing Enterobacteriaceae: considerations for diagnosis, prevention and drug treatment. Drugs 2003; 63: 353-65.

18.- Firman G. Sistema de clasificación de severidad de enfermedad APACHE II. www.intermedicina.com/Avances/Clinica/ ACL68.htm. (accedido marzo 2008).

19. - Rello J, Ollendorf D A, Oster G, VeraLlonch M, Bellm L, Redman R, et al. Epidemiology and outcomes of ventilatorassociated pneumonia in a large US database. Chest 2002; 122: 2115-21.

20.- Indicadores de infección intrahospitalaria. Ministerio de Salud, Gobierno de Chile. www.minsal.cl/ici/S_1/U_14/Indicadores\% 202004.pdf. (accedido marzo 2008).

21.- Informe IIH 2005: 30; Ministerio de Salud.
Gobierno de Chile. www.minsal.cl/ici/S. (accedido marzo 2008).

22.- UNESCO. Non-metric Multidimensional Scaling. www.unesco.org/webworld/idams/ advguide/Chapt8. (accedido diciembre 2008).

23.- CDC. Guidelines for Prevention of Nosocomial Pneumonia. MMWR Morb Mortal Wkly Rep 1997; 46 (RR-1): 1-79 www.cdc.gov/mmwr/preview/mmwrhtml/. (accedido noviembre 2008).

24.- Noor A, Hussain S F. Risk factors associated with development of ventilator associated pneumonia. J Coll Physicians Surg Pak 2005; 15: 92-5.

25.- Jordà M R, Torres M A, Ariza F J, Álvarez F, Barcenilla F. Recomendaciones para el tratamiento de la neumonía intrahospitalaria grave. Arch Bronconeumol 2004; 40: 518-33.

26.- Koulenti D, Myrianthefs P, Dimopoulos G, Baltopoulos G. Hospital-acquired pneumonia caused by methicillin-resistant Staphylococcus aureus. Enferm Infecc Microbiol Clin 2005; 23 Suppl 3: 37-45.

27. - Álvarez A, Bavestrello L, Labarca J M, Calvo A. Tratamiento de la neumonía asociada a ventilación mecánica. Rev Chil Infect 2001; 18 (Supl 2): S58-65.

28.- Ruiz M, Guerrero J, Romero C. Etiología de la neumonía asociada a ventilación mecánica en un hospital clínico: Asociación con co-morbilidad, uso previo de antimicrobianos y mortalidad. Rev Chil Infect 2007; 24: 131-6.

29.- Chastre J. Evolving problems with resistant pathogens. Clin Microbiol Infect 2008; 14 Suppl 3: 3-14.

30.- Slama T G. Gram-negative antibiotic resistance: there is a price to pay. Crit Care 2008; 12 Suppl 4: S4.

31.- Vila J, Pachón J. Therapeutic options for Acinetobacter baumannii infections. Expert Opin Pharmacother 2008; 9: 587-99.

32.- Díaz E, Muñoz E, Agbaht K, Rello J. Management of ventilator-associated pneumonia caused by multiresistant bacteria. Curr Opin Crit Care 2007; 13: 45-50. 